\title{
Dislocation Slip Behavior of Ni Single Crystal Under Dynamic Compression
}

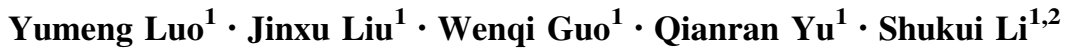

Received: 21 January 2016/ Accepted: 7 March 2016/Published online: 15 March 2016

(c) Society for Experimental Mechanics, Inc 2016

\begin{abstract}
Face-centered-cubic $\mathrm{Ni}$ single crystal was compressed along [011] direction at a strain rate of $10^{3} \mathrm{~s}^{-1}$, and microscopic characterization of slip bands was analyzed to investigate dynamical deformation mechanism of the Ni single crystal. By analyzing Schmid Factor and orientation of slip bands, the specific slip planes were revealed. Furthermore, by analysis of Schmid Factors and dimensions of the deformed sample, the slip directions are revealed, and the ratio of the slipping-induced displacement (D-ratio) related to each slip system were determined. For comparison, quasi-static compression (at a strain rate of $10^{-3} \mathrm{~s}^{-1}$ ) along [011] direction was conducted to a same $\mathrm{Ni}$ single crystal sample. Results show that the dynamic deformation process (at a strain rate of $10^{3} \mathrm{~s}^{-1}$ ) of $\mathrm{Ni}$ single crystal is accomplished via dislocation slip, and the activated slip systems under dynamic loading are proved to be (111) [1110], (111) [1̄01], (111) [110] and (111) [101]. Moreover, the quasi-static compression experimental results show the same slip systems, and the calculated slipping-induced displacement related to above 4 slip system have a ratio of nearly 1:3.8:3.8:1 under quasi-static compression. Thus, in a considerable strain rate range from $10^{-3}$ to $10^{3} \mathrm{~s}^{-1}$, the activated slip systems and the ratio of their corresponding slipping-induced displacement are the same. However, experimental results show that, the slip
\end{abstract}

Jinxu Liu

liujinxu@bit.edu.cn

1 School of Material Science and Engineering, Beijing Institute of Technology (BIT), No. 5 Yard, Zhong Guan Cun South Street, Beijing 100081, China

2 State Key Laboratory of Explosion Science and Technology, Beijing Institute of Technology (BIT), No. 5 Yard, Zhong Guan Cun South Street, Beijing 100081, China bands on side surface of $\mathrm{Ni}$ single crystal subjected to dynamic compression is denser than that of the quasi-static compressed sample, indicating that the slip bands are formed more easily under dynamic loading condition.

Keywords Single crystal - Dynamic plastic deformation · Dislocations $\cdot$ Nickel $\cdot$ Slip systems

\section{Introduction}

The plastic deformation behavior of single crystals has attracted much attention, for it is a good start to investigate materials with a simple microstructure to uncover the deformation behaviors and mechanisms. To date, extensive research papers about the quasi-static deformation behaviors and mechanisms of single crystals such as $\mathrm{Ag}$ [1], $\mathrm{Cu}$ [1-3], Nb [4, 5], Mo [6], Mg [7], Ti [8], Ni [9] and others [10] have been reported. Among these reports, a relatively clearer understanding of deformation mechanisms of single crystals under quasi-static loading condition has been obtained, and in-depth studies were carried out on the deformation inhomogeneity, microtexture development, strain hardening together with the formation and development of bands. However, in the field of dynamic deformation of single crystals, though there are lots of simulation studies [11-14], experimental researches are relatively less. According to the results, it seems that the extant of strain rate effect of single crystals with various structures are significantly different. For instance, Ta, Mo and $\mathrm{Ni}$ single crystals are reported to show a really unobvious strain rate effect on dislocation nucleation $[15,16]$, but $\mathrm{Al}$ [17] exhibits a strong strain rate effect on mechanical properties, while the dominate deformation mechanism of $\mathrm{Mg}$ [18] is greatly influenced by strain rate. 
Thus, strain rate may play a great role in deformation process, and the dynamic deformation of single crystal may have significant difference with the quasi-static one. However, based on the studies mentioned before, the existent researches about the dynamic deformation of single crystals are mostly focused on the strain rate effect of mechanical properties or competition between twinning and dislocation slip, but studies about dynamic deformation mechanism and the relative slip systems or twinning systems are relatively less. Since the research about dynamic deformation mechanism of single crystals plays an important role in thoroughly reveal and predict the deformation mechanisms of polycrystalline materials, in this work, Ni single crystal, which owns a typical FCC crystal structure, is select for investigation.

$\mathrm{Ni}$ is a FCC metal material with good ductility, and $\mathrm{Ni}$ alloy is widely used as structure material in high temperature environment. In the field of deformation process of $\mathrm{Ni}$ and Ni-based single crystal, lots of Refs. [19-22] are focus on Ni-based super-alloy single crystal, but researches [9, $23,24]$ about FCC Ni single crystal are relatively less. Owing to the completely different crystal structures, the deformation mechanism of FCC Ni single crystal is greatly different from that of Ni-based super-alloy single crystal, and the dynamic deformation mechanism of FCC Ni single crystal still lacks a systematically study. Grain fragmentation have been observed in polycrystalline $\mathrm{Ni}$ deformed under high strain rate [25], but no experimental study reports the dynamic deformation process or mechanism of FCC Ni single crystal. In previous works about the deformation mechanisms of FCC Ni single crystals, attention has been directed towards the quasi-static deformation. In this field, Lychagin et al. [9] investigated the regularities of macro- and mecoscale deformation of [111] nickel single crystals with different shapes, and then determined the strain localization factors. According to his research, the regions of deformation macrobands and folds are not localized in the area having the most of strain, and the deformation of specimen is inhomogeneous. However, the formation plane and direction of the macrobands, folds and shear traces were not explicitly pointed out. Besides, Chen [23] investigated the saturated dislocation structures of cyclic deformed $\mathrm{Ni}$ single crystals. According to his research, when the quasi-static load was applied along [110] direction, the slip systems calculated by slip trace

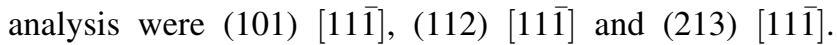
Similarly, in the investigation about pre-straining effect in $\mathrm{Ni}$ single crystal made by El-Awady et al. [24], slip systems activated under quasi-static compression were proved

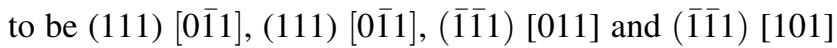
by analyzing Schmid Factor. Thus, previous works about FCC Ni single crystals have proved that the deformation mechanism under quasi-static loading condition is dislocation slip, and a definite analysis of slip systems was given, but the slipping-induced displacement related to each slip system is not analyzed. In the past studies, the activated slip systems can be proved, but whether the activated slip systems have the same contribution to deformation or not have not been taken seriously. In other words, till now, the ratio of the slipping-induced displacement (named as "D-ratio" for short) related to each slip system is still not clear. Apart from the unsettled question of the quasi-static deformation process of FCC Ni single crystals, the dynamic deformation mechanism of $\mathrm{Ni}$ is unknown because of the lack of experimental study.

In conclusion, several problems towards the deformation mechanisms of Ni single crystal remain unsettled. Firstly, whether dislocation slip is still the deformation mechanism of Ni single crystal under high strain rate? Secondly, even if dislocation slip is the deformation mechanism, it is not sure that the specific activated slip systems and their D-ratio under dynamic loading are the same as that under quasi-static condition.

In the present paper, FCC Ni single crystal was compressed along [011] direction at a strain rate of $10^{3} \mathrm{~s}^{-1}$, so as to investigate the dynamic deformation mechanism and behavior. For comparison, quasi-static loading (at a strain rate of $10^{3} \mathrm{~s}^{-1}$ ) along [011] direction with a same strain is also conducted. Then the slip systems and their corresponding D-ratio are analyzed according to the deformed dimensions of sample and orientations of slip bands. Furthermore, the different feature of slip bands under different strain rate condition is discussed.

\section{Experimental}

FCC Ni single crystal samples with a purity of $99.99 \%$ were sectioned into 3 same cubes followed by electropolishing. Before compression, EBSD (Electron Backscattered Diffraction) was carried out using a field emission gun scanning electron microscope HITACHI S4800 on the side face for orientation analysis. The EBSD data was analyzed using the commercially available TexSem Laboratory's OIM software. Then, Fig. 1a, b show the lateral view and top view of the original FCC Ni single crystal sample with a dimension of $5 \times 5 \times 5 \mathrm{~mm}$, and Fig. 1d, e are the inverse pole figure (IPF) and image quality (IQ) with a step size of $50 \mathrm{~nm}$ of a side surface as illustrated in Fig. 1c. From Fig. 1d, e, it can be confirmed that the sample has a single-crystal structure, and the crystal orientation of the three mutually perpendicular

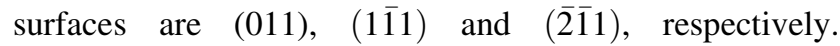
According to the surface orientation of the cubic sample, a 
particular three dimensional coordinate system for sample is established. The origin of this sample coordinate is selected to be Vertex $\mathrm{O}$, and the Axes of the sample coordinate are perpendicular to the surfaces of cubic sample. Thus, the $\mathrm{X}^{\prime}, \mathrm{Y}^{\prime}$ and $\mathrm{Z}^{\prime}$ Axes are parallel to $[\overline{2} \overline{1} 1]$ [111], and [011] direction of the crystal, respectively, as shown in Fig. 1c.

Dynamic compression experiment was conducted to a Ni single crystal along $Z^{\prime}$ Axis of the sample coordinate ([011] direction of the crystal coordinate) utilizing a Split Hopkinson Pressure Bar (SHPB) at room temperature, and the schematic sketch of SHPB is illustrated in Fig. 2a. The strain rate was about $3000 \mathrm{~s}^{-1}$, and the strain was controlled by using a stopper ring, as shown in Fig. 2b. During the first round of loading pulse of SHPB experiment, the sample deforms to the same height of the stopper ring, and the stopper ring begins to bear the loading. After that, the stopper ring can prevent the sample from a subsequent deformation and ensure that the sample was compressed only once. In this work, the strain of the first single crystal sample was controlled to be 0.4 . In order to investigate whether the slip systems and their corresponding D-ratio are influenced by the strain, a dynamic compression experiment was conducted along [011] to a same Ni single crystal until strain reached 0.12 . For comparison, a quasistatic compression loading was applied to the third single crystal sample along the same direction of [011] until strain reached 0.4 at strain rate of $10^{-3} \mathrm{~s}^{-1}$. Since all the surfaces of samples were electro-polished, the orientation analysis of deformed sample can be taken directly by EBSD, and the slip bands on the side surface were observed by SEM.

According to SEM micrographs of the slip bands on the side faces, the specific slip planes were analyzed. It is necessary to note that all of the calculations are based on the theory that two slip bands located in two adjacent sides respectively form only one plane. This specific plane is the slip plane. The detailed method for analyzing slip planes is illustrated below. Figure $3 \mathrm{a}$ is a sketch of slip bands on two side faces of the deformed sample, and the relationship between slip bands and the particular 3D Coordinate System of the sample is shown in Fig. 3b. If the normal vector of the slip plane is $\vec{p}$, the geometrical relationship between
Fig. 1 Macroscopic photographs and the EBSD testing results of FCC Ni single crystal before deformation. a Lateral view of the specimen; b Top view of the specimen; c Diagrammatic sketch of sample coordinate and the compression loading condition; d Inverse pole figure of the surface marked yellow in (c); e IPF and IQ of the surface marked yellow in (c) (Color figure online)
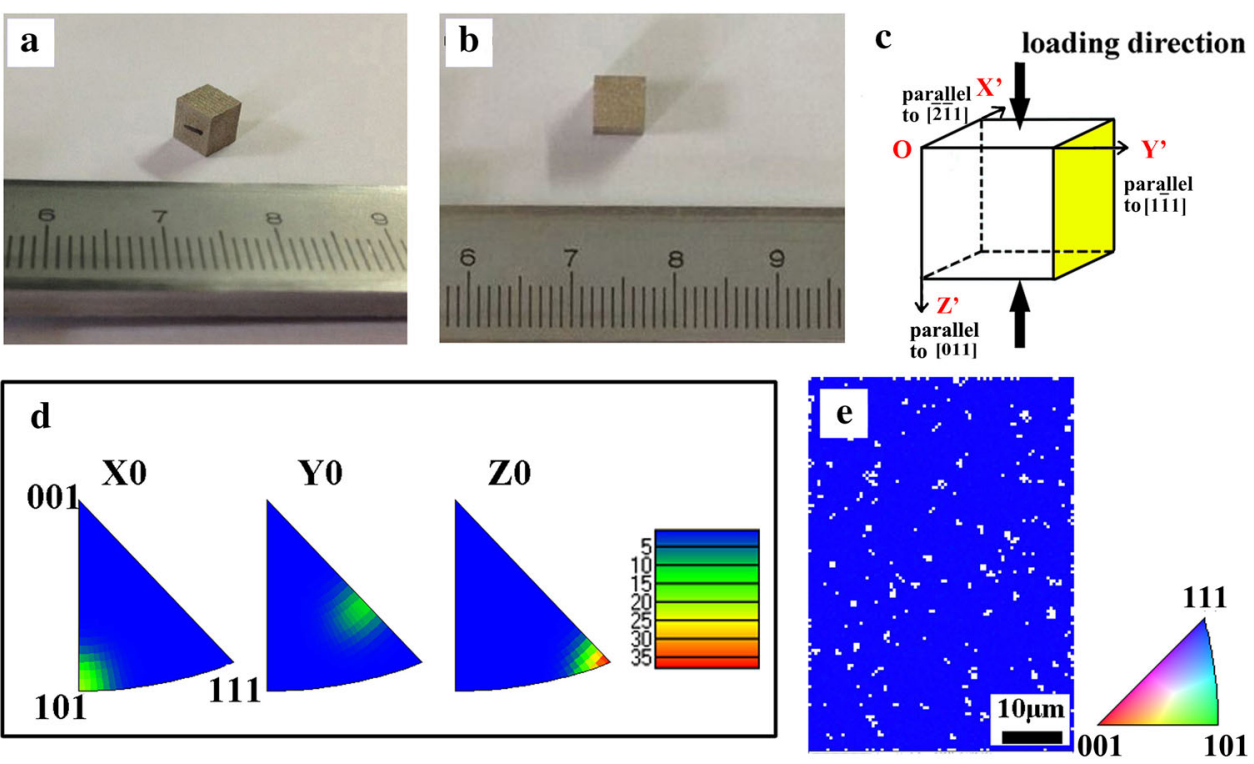

Fig. 2 Schematic sketch of Split Hopkinson pressure bar equipment and strain controlling method. a Split Hopkinson pressure bar and $\mathbf{b}$ Stopper ring

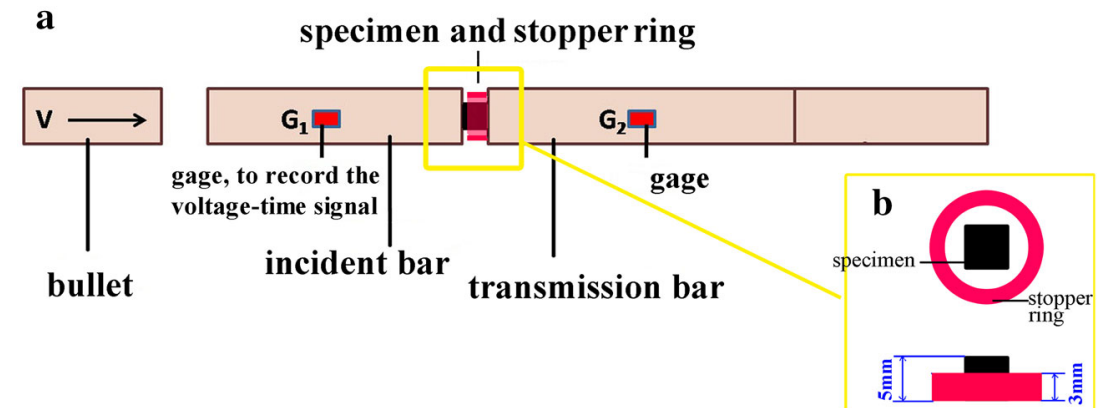


Fig. 3 Sketch of slip face analyses. a Diagrammatic sketch of slip bands on side face of single crystal after deformation; b Orientation relationship of slip bands on adjacent side faces $\mathbf{a}$

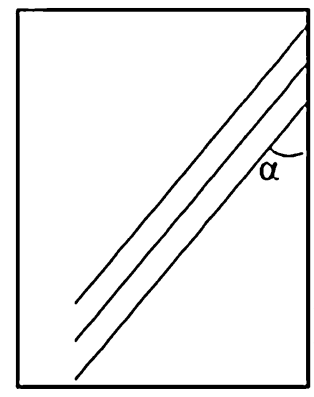

b

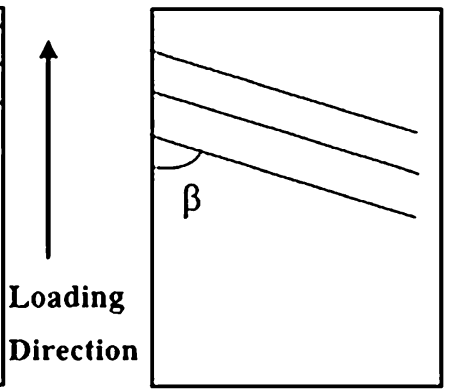

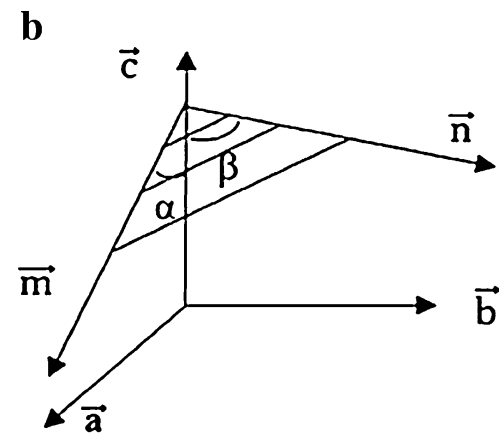

the slip plane and slip bands in Fig. 3 can be deduced as follows:

$\left\{\begin{array}{c}\cos (\pi-\alpha)=\frac{\overrightarrow{\mathrm{m}} \cdot \overrightarrow{\mathrm{c}}}{|\overrightarrow{\mathrm{m}}||\overrightarrow{\mathrm{c}}|} \\ \overrightarrow{\mathrm{m}} \cdot \overrightarrow{\mathrm{b}}=0\end{array}\right.$
$\left\{\begin{array}{c}\cos (\pi-\beta)=\frac{\overrightarrow{\mathrm{n}} \cdot \overrightarrow{\mathrm{c}}}{|\overrightarrow{\mathrm{n}}||\overrightarrow{\mathrm{c}}|} \\ \overrightarrow{\mathrm{n}} \cdot \overrightarrow{\mathrm{a}}=0\end{array}\right.$

$\left\{\begin{array}{l}\overrightarrow{\mathrm{m}} \cdot \overrightarrow{\mathrm{p}}=0 \\ \overrightarrow{\mathrm{n}} \cdot \overrightarrow{\mathrm{p}}=0\end{array}\right.$

Using formula (1)-(3), the slip plane can be identified. It should be noted that, because the slip bands on the side surface show a step feature, the measurement of $\alpha$ and $\beta$ in Fig. 3a must be taken on the step instead of orientation of the whole slip band. Then, the slip direction and the D-ratio of each slip system are determined by a comprehensive analysis including Schmid Factor and the changes of sample dimensions after deformation.

\section{Results and Discussion}

\section{The Dynamic Deformation Mechanism}

After dynamic compression, the single crystal sample deforms into right parallelepiped, as shown in Fig. 4. The dimension of the deformed sample is illustrated in Fig. 5. It $\mathbf{a}$

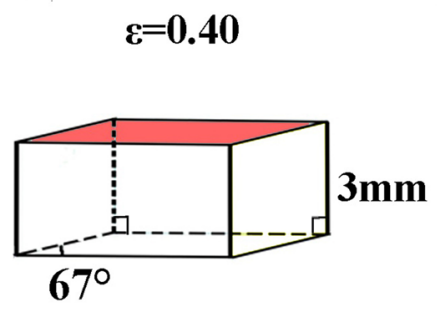

b

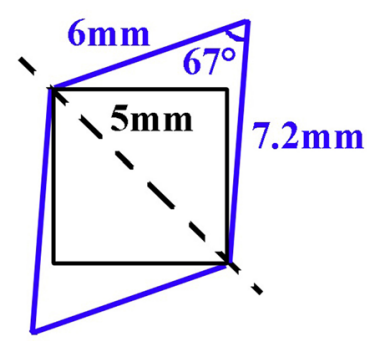

Fig. 5 Dimensions of Ni single crystal after dynamic compression with a strain of 0.4. a Lateral view and $\mathbf{b}$ Top view

is obviously that the loading surface of the sample (marked as red in Fig. 5a) turned into parallelogram, and the deformed sample was symmetric about the loading direction. Moreover, EBSD results show that the deformed sample keeps a single crystal structure, and the measuring face has same crystal orientations as the original one. Furthermore, according to EBSD results, there is no twinning observed in the deformed crystal, indicating that twinning is not the deformation mechanism.

As mentioned before, the polished surfaces of the sample were observed by SEM after dynamic compression. Figure 6 is the SEM photographs and diagrammatic sketch of the slip bands on the side surfaces of the dynamically deformed $\mathrm{Ni}$ single crystal. As shown in Fig. 6b, e, there are a large number of slip bands densely spreading on both (111) and $(\overline{2} \overline{1} 1)$ side surfaces. Thus, dislocation slip is proved to be the deformation mechanism of Ni single crystal.

Fig. 4 Macroscopic photographs of Ni single crystal after dynamic deformation with a stain of 0.4, and the EBSD testing results. a Top view; b Lateral view; c Inverse pole figure of the (1111) side surface; d IPF and IQ of the (11̄1) side surface
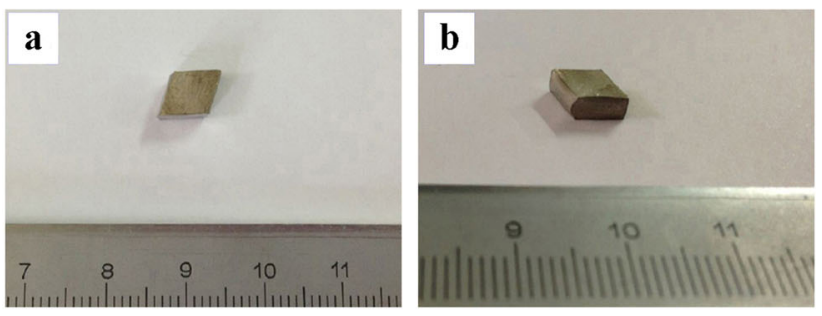
Fig. 6 SEM photograph and diagrammatic sketch of slip bands on the side faces of $\mathrm{Ni}$ single crystal after dynamic deformation. a Diagrammatic sketch of loading, the marked face is (1 $\overline{1} 1)$; b Slip bands on the (11̄1) side surface; c Diagrammatic sketch of slip bands on (1111) plane; d Diagrammatic sketch of loading, the marked face is $(\overline{2} \overline{1} 1)$; e Slip bands on the $(\overline{2} \overline{1} 1)$ side surface; $\mathbf{f}$ Diagrammatic sketch of slip bands on $(\overline{2} \overline{1} 1)$ plane a

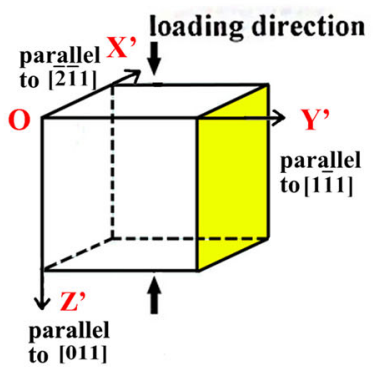

d

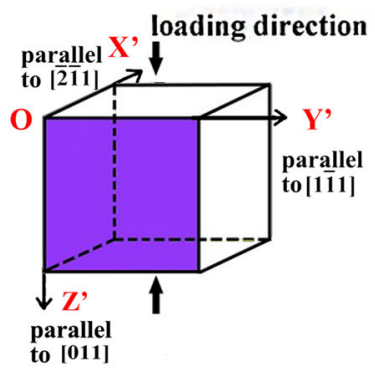

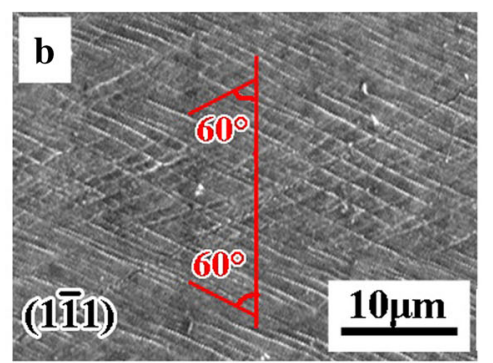

c (11̄1)
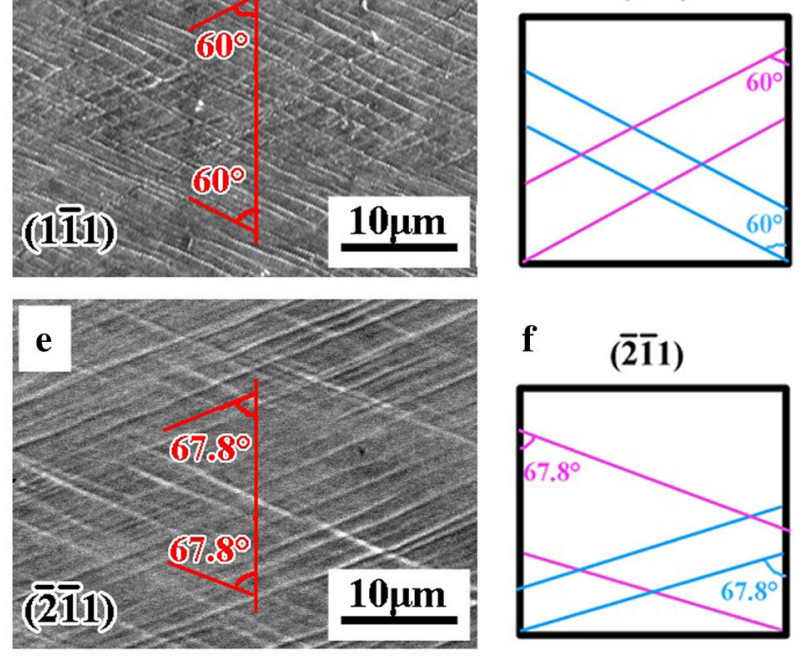

\section{The Analysis of Slip System}

It is well known that $\mathrm{Ni}$ is apt to deform along $\{111\}\langle 110\rangle$ at strain rate of $10^{-3} \mathrm{~s}^{-1}$ [26], and other research proved that, $1 / 6\{111\}\langle 112\rangle$ and $\{111\}\langle 110\rangle$ are the easiest nucleation slip systems of $\mathrm{Ni}$ single crystal. Thus, $1 / 6\{111\}\langle 112\rangle$ and $\{111\}\langle 110\rangle$ are considered to be the slip system under $10^{-3} \mathrm{~s}^{-1}$ loading condition. In this research, the strain rate of compression loading is $10^{3} \mathrm{~s}^{-1}$, and the slip system of $\mathrm{Ni}$ single crystal under high strain rate may change.

Figure $6 \mathrm{~b}$ is the SEM photograph of the (11̄1) side surface of the dynamic deformed specimen (marked in yellow in Fig. 6a). It is obviously that there are two groups of slip bands on the (1111) side surface along different orientations. According to a statistic study, all of the slip bands are at nearly $60^{\circ}$ to the loading direction. Similarly, two groups of slip bands are observed on the $(\overline{2} \overline{1} 1)$ side surface (marked in purple in Fig. 6e), and the angle between slip bands and loading direction ([011]) is about $67.8^{\circ}$, as shown in Fig. 6f. As stated before, two slip bands located in two adjacent sides respectively can form only one specific plane, which is the slip plane. Combined with formula (1)-(3), the calculated slip planes are (111) and (111).

In this research, compression loading was applied along [011] direction. To analyze the slip direction, all the Schmid Factors of both $\{111\}\langle 112\rangle$ and $\{111\}\langle 110\rangle$ slip systems are taken into consideration. The analysis of Schmid Factor for above two slip systems shows that the Schmid Factor of (111) [110], (111) [101], (111) [110] and (111) [101] are $\frac{\sqrt{6}}{6}$, while the factor of (111) [ $\overline{2} 11]$ and (111) [211] are $\frac{\sqrt{2}}{3}$. Thus, all these 6 slip systems have a probability to be activated. As can be seen that the slip planes of above 6 slip systems belong to (111) and (111), which are corresponding to the slip planes analyzed by experimental results in the present work. However, the slip direction and the D-ratio of the slip systems are still unclear. Therefore, the next work is analyzing the direction of the dislocation slip system and the D-ratio of slip systems.

The direction of slip system is analyzed by dimension changes between the original and deformed sample. For convenience, all the crystallography vectors should be transformed into the sample coordinate system. The relationship between crystallography coordinate system $\left(\mathrm{X}_{0} \mathrm{Y}_{0} \mathrm{Z}_{0}\right)$ and sample coordinate system $\left(\mathrm{X}^{\prime} \mathrm{Y}^{\prime} \mathrm{Z}^{\prime}\right)$ is shown in Fig. 7. The $\mathrm{X}_{0}, \mathrm{Y}_{0}$ and $\mathrm{Z}_{0}$ Axis are parallel to the [100], [010], and [001] direction in the crystallography coordinate system, respectively. Accordingly, the $\mathrm{X}^{\prime}$, $\mathrm{Y}^{\prime}$, axis and $\mathrm{Z}^{\prime}$ axis in sample coordinate system is parallel to the $[\overline{2} \overline{1} 1],[1 \overline{1} 1]$ and [011] direction in crystallography coordinate system, respectively. The angles from $\mathrm{X}_{0}$ Axis to $\mathrm{X}^{\prime}$ axis, $\mathrm{Y}^{\prime}$ axis and $\mathrm{Z}^{\prime}$ axis are named as $\alpha_{1}, \beta_{1}$ and $\gamma_{1}$, respectively. Similarly, the angle from $Y_{0}$ Axis to the three Axes of the sample coordinate system are named as $\alpha_{2}, \beta_{2}$, and $\gamma_{2}$, while the angle from $Z_{0}$ Axis to the three Axes of the sample coordinate system are named as $\alpha_{3}, \beta_{3}$, and $\gamma_{3}$, respectively. According to the mathematical method for transformation of coordinate, in order to transform a crystallography vector into the sample coordinate system $\left(\mathrm{X}^{\prime} \mathrm{Y}^{\prime} \mathrm{Z}^{\prime}\right)$, the vector should plus the matrix $\left[\begin{array}{ccc}\cos \alpha_{1} & \cos \beta_{1} & \cos \gamma_{1} \\ \cos \alpha_{2} & \cos \beta_{2} & \cos \gamma_{2} \\ \cos \alpha_{3} & \cos \beta_{3} & \cos \gamma_{3}\end{array}\right]$, where $\left[\begin{array}{lll}\cos \alpha_{1} & \cos \beta_{1} & \cos \gamma_{1}\end{array}\right] \quad$ is $[-2-11] /$ $\sqrt{(-2)^{2}+(-1)^{2}+(1)^{2}}, \quad\left[\begin{array}{lll}\cos \alpha_{2} & \cos \beta_{2} & \cos \gamma_{2}\end{array}\right] \quad$ is 


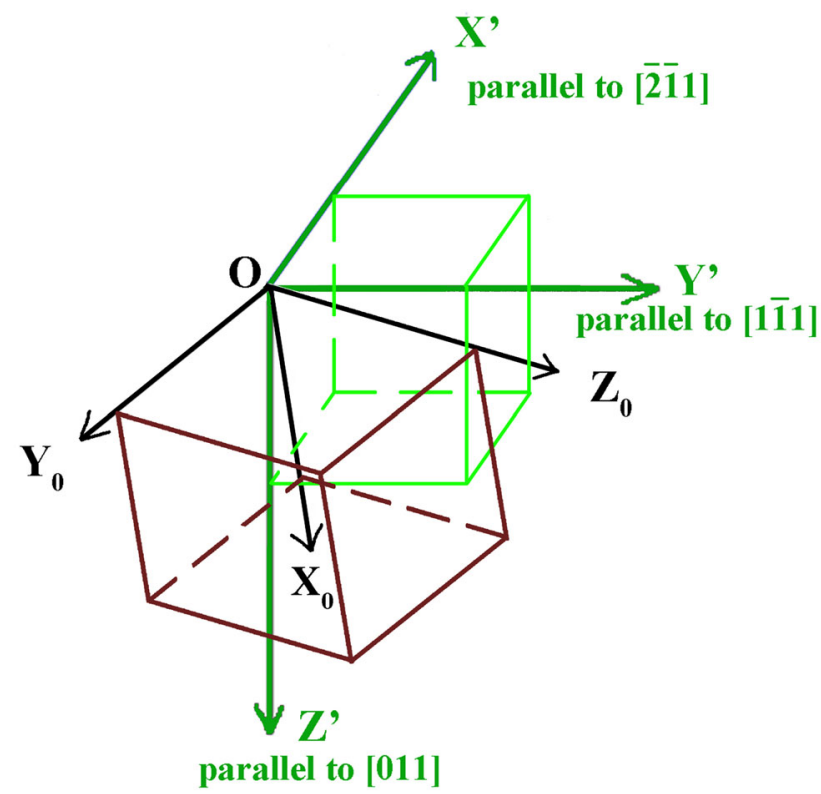

Fig. 7 The relationship between crystallography coordinate system $\left(\mathrm{X}_{0} \mathrm{Y}_{0} \mathrm{Z}_{0}\right)$ and sample coordinate system $\left(\mathrm{X}^{\prime} \mathrm{Y}^{\prime} \mathrm{Z}^{\prime}\right)$

$$
\begin{aligned}
& {[1-11] / \sqrt{(1)^{2}+(-1)^{2}+(1)^{2}} \text { and }\left[\begin{array}{llll}
\cos \alpha_{3} & \cos \beta_{3} & \cos
\end{array}\right.} \\
& \left.\gamma_{3}\right] \text { is }[011] / \sqrt{(0)^{2}+(1)^{2}+(1)^{2}} \text {. }
\end{aligned}
$$

For the sample coordinate system established in this research, the specific matrix $\left[\begin{array}{ccc}\cos \alpha_{1} & \cos \beta_{1} & \cos \gamma_{1} \\ \cos \alpha_{2} & \cos \beta_{2} & \cos \gamma_{2} \\ \cos \alpha_{3} & \cos \beta_{3} & \cos \gamma_{3}\end{array}\right]$ is

$$
\left[\begin{array}{ccc}
-\frac{2}{\sqrt{6}} & -\frac{1}{\sqrt{6}} & \frac{1}{\sqrt{6}} \\
\frac{1}{\sqrt{3}} & -\frac{1}{\sqrt{3}} & \frac{1}{\sqrt{3}} \\
0 & \frac{1}{\sqrt{2}} & \frac{1}{\sqrt{2}}
\end{array}\right] .
$$

Then all the crystallography

vectors occurred in the slip systems can transform into vectors in the sample system.

Due to the largest Shimid Factor, the slip systems (111)

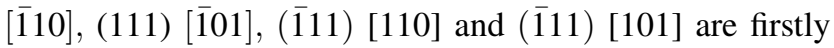
taken into consideration. Suppose that, when the activation of slip system (111) [1110] makes a relative displacement with a length of 1 along [110], the resulting relative displacement along $\mathrm{X}^{\prime}$ axis in the sample coordinate system is named as $X_{1}$, while $Y_{1}$ is named as the relative displacement along $\mathrm{Y}^{\prime}$ axis, and $\mathrm{Z}_{1}$ is named as the relative displacement along $Z^{\prime}$ axis. As a result of unit length displacement along [011] within (111) [110], the displacement vector $\left[\begin{array}{c}X_{1} \\ Y_{1} \\ Z_{1}\end{array}\right]$ in sample coordinate system $\left(\mathrm{X}^{\prime}\right.$ $\mathrm{Y}^{\prime} \mathrm{Z}^{\prime}$ ) is calculated by Eq. (4).

$$
\begin{aligned}
{\left[\begin{array}{l}
X_{1} \\
Y_{1} \\
Z_{1}
\end{array}\right]=} & {\left[\begin{array}{ccc}
-\frac{2}{\sqrt{6}} & -\frac{1}{\sqrt{6}} & \frac{1}{\sqrt{6}} \\
\frac{1}{\sqrt{3}} & -\frac{1}{\sqrt{3}} & \frac{1}{\sqrt{3}} \\
0 & \frac{1}{\sqrt{2}} & \frac{1}{\sqrt{2}}
\end{array}\right] \times\left[\begin{array}{c}
-1 \\
1 \\
0
\end{array}\right] } \\
& \times \frac{1}{\sqrt{(-1)^{2}+(1)^{2}+(0)^{2}}}
\end{aligned}
$$

Results show that, the displacement vector caused by the unit length displacement along [110] within (111) [ $\overline{1} 10]$ is $\left[\begin{array}{c}\frac{\sqrt{3}}{6} \\ -\frac{\sqrt{6}}{3} \\ \frac{1}{2}\end{array}\right]$ in the sample coordinate system. Similarly, the displacement vectors caused by the unit length displacement within (111) [101], (111) [110] and (111) [101] are calculated to be

$$
\begin{aligned}
{\left[\begin{array}{l}
X_{2} \\
Y_{2} \\
Z_{2}
\end{array}\right]=} & {\left[\begin{array}{c}
\frac{\sqrt{3}}{2} \\
0 \\
\frac{1}{2}
\end{array}\right],\left[\begin{array}{l}
X_{3} \\
Y_{3} \\
Z_{3}
\end{array}\right]=\left[\begin{array}{c}
-\frac{\sqrt{3}}{2} \\
0 \\
\frac{1}{2}
\end{array}\right] \text { and }\left[\begin{array}{l}
X_{4} \\
Y_{4} \\
Z_{4}
\end{array}\right] } \\
= & {\left[\begin{array}{c}
-\frac{\sqrt{3}}{6} \\
\frac{\sqrt{6}}{3} \\
\frac{1}{2}
\end{array}\right] }
\end{aligned}
$$

respectively.

In this work, the deformation process is under a planar constraint condition. Due to the friction on the loading surface, deformation between the center part and surface of the sample may have some differences. We select point $\mathrm{O}$ to be the origin point of the sample coordinate system, and point $\mathrm{A}$ is selected to make a general analysis of the slip systems and their corresponding D-ratio, as illustrated in Fig. 8. For the selected point A of the sample, the displacement vector in the sample coordinate system is named as $\left[\begin{array}{c}X \\ Y \\ Z\end{array}\right]$. Suppose that activation of slip system (111) [110] makes a relative displacement of " $t$ " in length, the displacement along $\mathrm{X}^{\prime}, \mathrm{Y}^{\prime}$ and $\mathrm{Z}^{\prime}$ Axis of the sample coordinate system can be calculated by $\mathrm{t} \times X_{1}, \mathrm{t} \times Y_{1}$ and $\mathrm{t} \times Z_{1} . X_{1}, Y_{1}$ and $Z_{1}$ have been calculated in Eq. (4). Similarly, it is supposed that the activation of slip system (111) $[\overline{101}]$ makes a displacement of " $u$ ", the relative displacements along three Axes of the sample coordinate 
system are $\mathrm{u} \times\left[\begin{array}{l}X_{2} \\ Y_{2} \\ Z_{2}\end{array}\right]$, while for "v" made by activation of (111) [110] and "w" made by activation of (111)[101], the relative displacements are $\mathrm{v} \times\left[\begin{array}{c}X_{3} \\ Y_{3} \\ Z_{3}\end{array}\right]$ and $\mathrm{w} \times\left[\begin{array}{c}X_{4} \\ Y_{4} \\ Z_{4}\end{array}\right]$, respectively. Therefore, u:v:w:t in fact presents the D-ratio of the 4 slip systems listed before. To point A, the displacement vector is

$\left\{\begin{array}{c}X=t \times X_{1}+u \times X_{2}+v \times X_{3}+w \times X_{4} \\ Y=t \times Y_{1}+u \times Y_{2}+v \times Y_{3}+w \times Y_{4} \\ Z=t \times Z_{1}+u \times Z_{2}+v \times Z_{3}+w \times Z_{4}\end{array}\right.$

According to the experimental result, when the sample was dynamic compressed with a strain of 0.4 , the measured displacement vector of point $\mathrm{A}$ in the sample coordinate system (as shown in Fig. 8) is $\left[\begin{array}{c}4.34 \\ 1.18 \\ 2\end{array}\right]$, and this displacement vector has to meet the following equation

$\left\{\begin{array}{l}4.34=t \times \frac{\sqrt{3}}{6}+u \times \frac{\sqrt{3}}{2}-v \times \frac{\sqrt{3}}{2}-w \times \frac{\sqrt{3}}{6} \\ 1.18=-t \times \frac{\sqrt{6}}{3}+w \times \frac{\sqrt{6}}{3} \\ 2=t \times \frac{1}{2}+u \times \frac{1}{2}+v \times \frac{1}{2}+w \times \frac{1}{2}\end{array}\right.$

Then, it can be calculated that

$\frac{(v-u)}{(t-w)} \approx 3.8$

As mentioned before, (111) [110] and (111) [101] have the same Schmid Factor. Moreover, when the activation of (111) [110] and (111) [101] cause the same length displacement, the relative displacements induced by the two slip systems along $\mathrm{Z}^{\prime}$ Axis are equal, and the relative displacements along $\mathrm{X}^{\prime}$ Axis $\left(\mathrm{X}_{1}\right)$ induced by (111) [110] are the opposite numbers to $\mathrm{X}_{4}$ induced by (111) [101], while $\mathrm{Y}_{1}$ are the opposite numbers to $\mathrm{Y}_{4}$, respectively. Thus, the slip directions of (111) [1110] and (111) [101] are symmetric with respect to the loading direction, as shown in

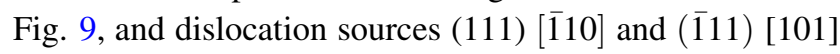
have the high order symmetry of both applied stress and crystallographic orientation of the single crystal with respect to the loading axis, leading to a same activated opportunity. Likewise, (111) [101] and (111) [110] have the same opportunity to activate. In other words,

$\left\{\begin{array}{l}|t|=|w| \\ |u|=|v|\end{array}\right.$

Combining Eq. (9) with Eq. (8), it can be calculated that

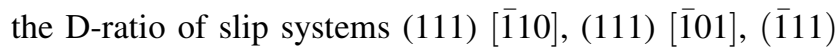
[110] and (111) [101] is nearly 1:3.8:3.8:1.

The same method is used to analyze whether (111) $(\overline{2} 11)$ and (111) [211] are the activated slip systems or not. It is obviously that (111) $(\overline{2} 11)$ and $(\overline{1} 11)$ [211] are symmetric about the loading direction and have the same Schmid Factor. Thus, (111) (211) and (111) [211] have the same opportunity to activate. However, for point $\mathrm{A}$ in Fig. 8, when the dislocation equally activate along (111) (211) and (111) [211], the displacement vector of point A cannot be achieved. Thus, slip systems (111) (211) and (111) [211] are not the activated dislocation slip systems.

In order to make sure that the slip systems and their corresponding D-ratio are not influenced by the strain, a Ni single crystal was dynamic compressed until strain reach to 0.12 along [011] at a strain rate of $10^{3} \mathrm{~s}^{-1}$. Figure 10 shows dislocation lines on the (111) side surface of the dynamic deformed sample with a strain of 0.12 and 0.4 . It can be observed that, when the strain is about 0.12 , there are less slip bands along two orientations, though one group of slip bands is not very clear. The angle between slip bands on the (11) 1 ) side surface and loading direction of the sample with a strain of 0.12 is $60^{\circ}$, and the angle

Fig. 8 Diagrammatic sketch of displacement of point A of the FCC Ni single crystal specimen with a strain of 0.4 . a Diagrammatic sketch of the specimen before and after dynamic deformation and b Dimensions of the loading surface after dynamic deformation

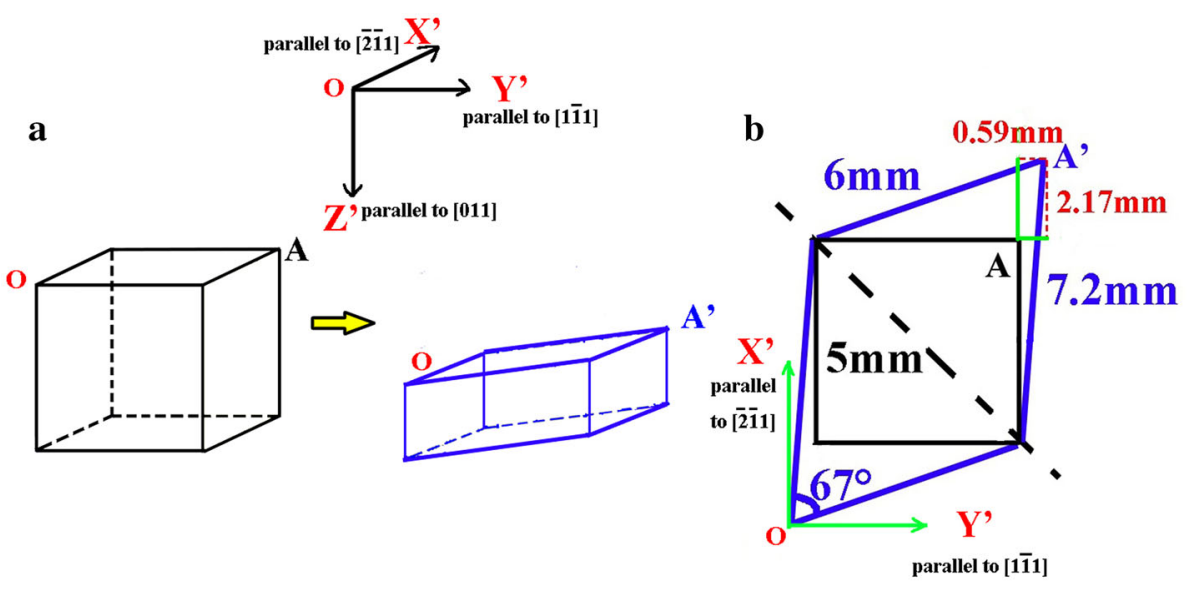




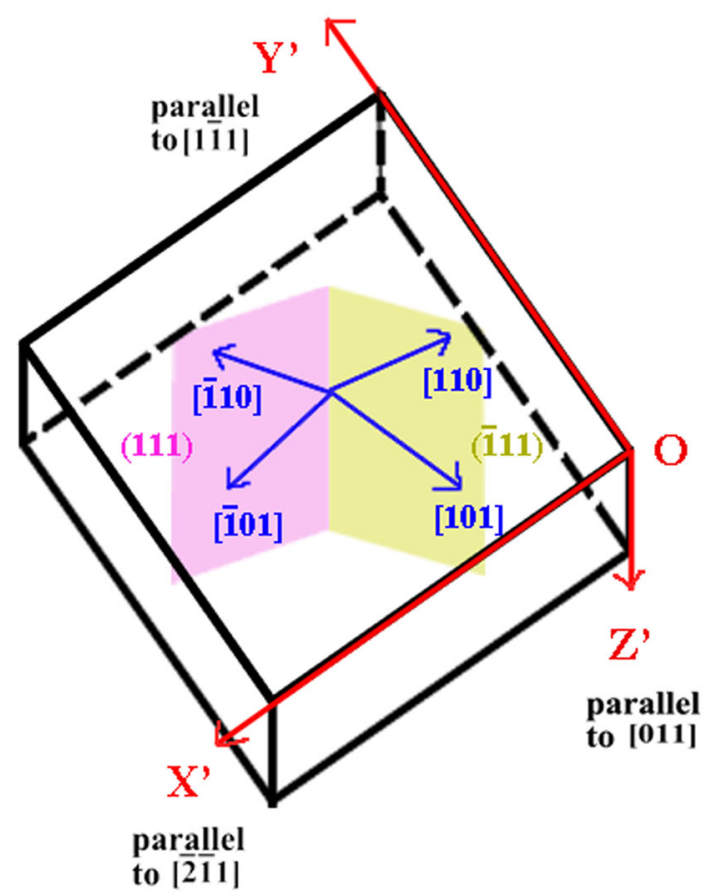

Fig. 9 Diagrammatic sketch of relationship between slip system

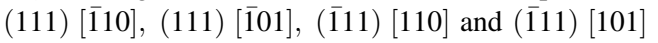

between slip bands on the ( $\overline{2} 11)$ side surface and loading direction is $67.8^{\circ}$, indicating that the dislocation slip planes of sample with a strain of 0.12 are the same as that with a strain of 0.4. Moreover, Fig. 11 shows the dimensions of the loading surface after dynamic deformation with a strain of 0.12 , and it is evidently that the sample is also symmetric about the loading direction. According to the

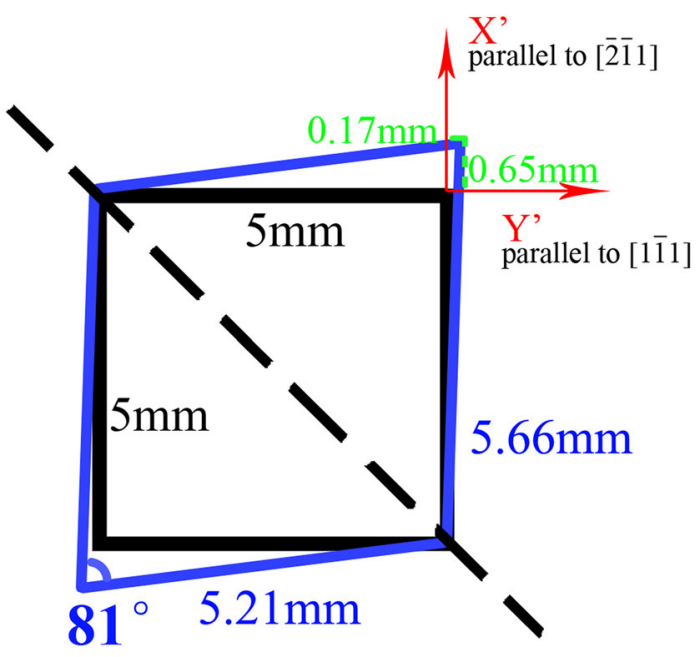

Fig. 11 Dimensions of the loading surface after dynamic deformation with a strain of 0.12

analysis of deformed dimension, it can be concluded that the slip system and their corresponding D-ratio of the sample with a strain of 0.12 are the same as that of 0.4 sample, showing the unaffected nature of the slip systems and their corresponding D-ratio by strain.

\section{Comparison Between Dynamic Loading Condition and Quasi-Static Loading Condition}

For comparison, quasi-static compression is applied to $\mathrm{Ni}$ single crystal along [011] direction until the strain reaches 0.4 . Figure 12 shows the macro-photo, inverse pole figure and EBSD results of the quasi-static deformed sample.
Fig. 10 Evolution process of the slip bands on the side surface of the specimen with different strain $(0,0.12$, and $0.4)$. a-c Evolution models at different strain $(0,0.12$, and 0.4). d-f SEM pictures of (11̄1) side surface at different strain $(0,0.12$ and 0.4$)$ $\mathbf{a}$ loading direction
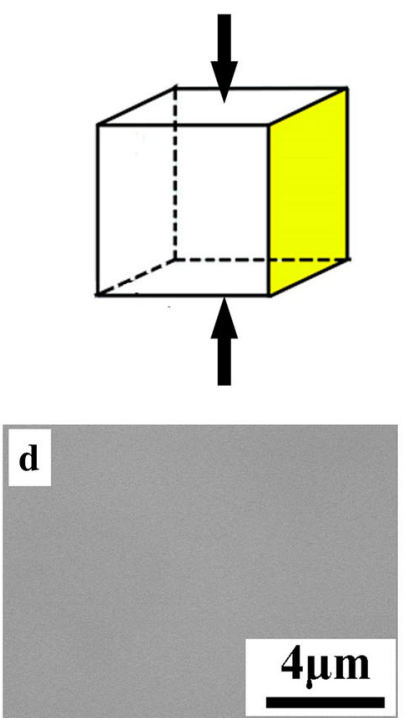

b
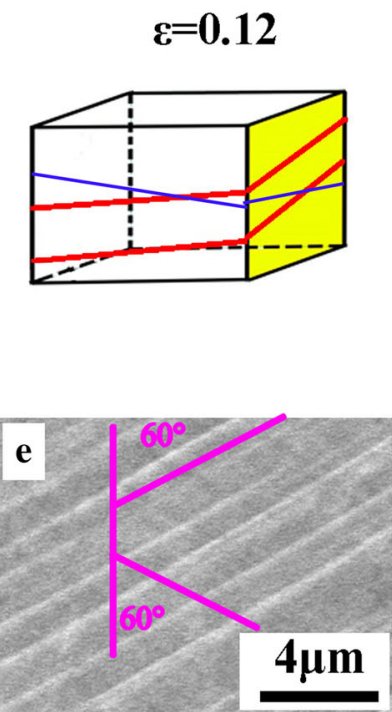
Fig. 12 Macroscopic

photographs of Ni single crystal after quasi-static deformation with a stain of 0.4 , and the EBSD testing results. a Top view; b Lateral view; c Inverse pole figure of the (1) 1$)$ side surface; and $\mathbf{d}$ IPF and IQ of the (11̄1) side surface
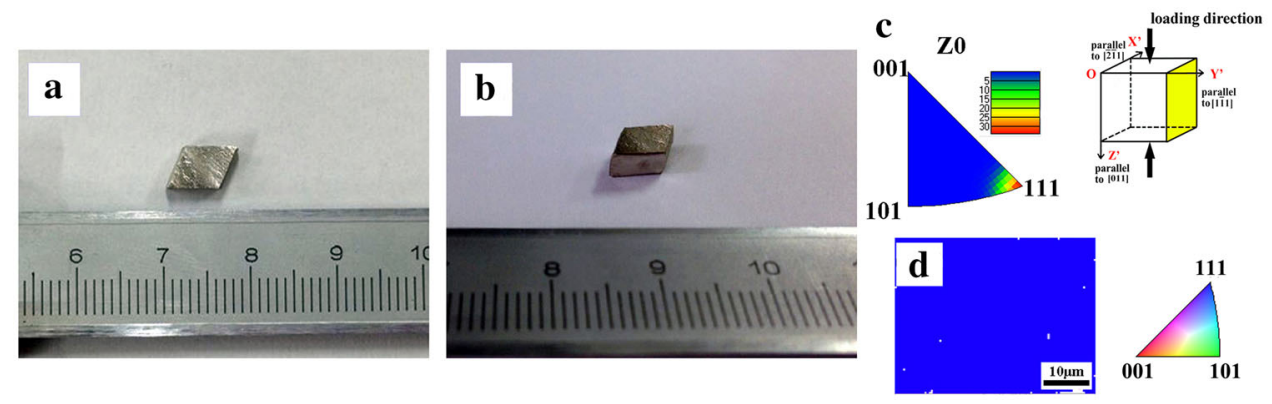
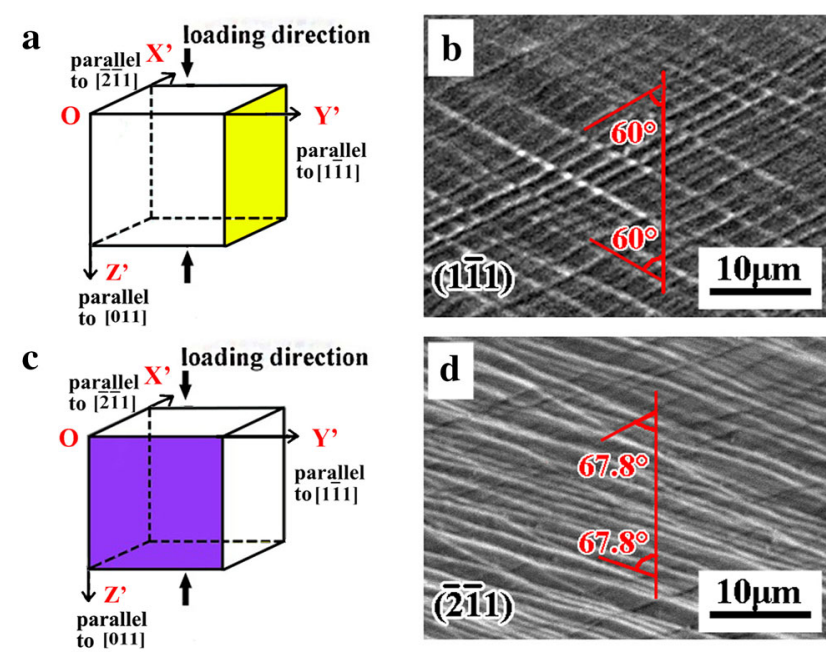

Fig. 13 SEM photograph of slip bands on the side faces of Ni single crystal after quasi-static loading. a Diagrammatic sketch of loading, the marked face is $(1 \overline{1} 1)$; b Slip bands on the (111) side surface; c Diagrammatic sketch of loading, the marked face is (2) $\overline{1} 1)$; and d Slip bands on the $(\overline{2} \overline{1} 1)$ side surface
It is evidently that the sample remains a single crystal structure after quasi-static compression. It can be observed that, the crystal orientation of the quasi-static deformed crystal is corresponding to original sample. Moreover, the dimension of the quasi-static deformed sample is the same as that of dynamic deformed sample.

Figure 13 shows the slip bands formed on the side surface of the quasi-static deformed sample. The slip bands have the same orientations as that formed under dynamic loading condition, which are nearly $60^{\circ}$ with the loading axis on the (111) side face and about $67.8^{\circ}$ with the loading axis on the $(\overline{2} \overline{1} 1)$ side surface. Therefore, under quasi-static condition, FCC Ni single crystal deforms by the same slip planes (111) and (111) as that under dynamic compression condition. Moreover, the quasi-static deformed sample has the same dimensions as that subjected to dynamic compression. Thus it can be concluded that the sample deforms along the same slip direction and has a same D-ratio of slip systems as that under dynamic compression. However, when further analysis is done, some distinctions are
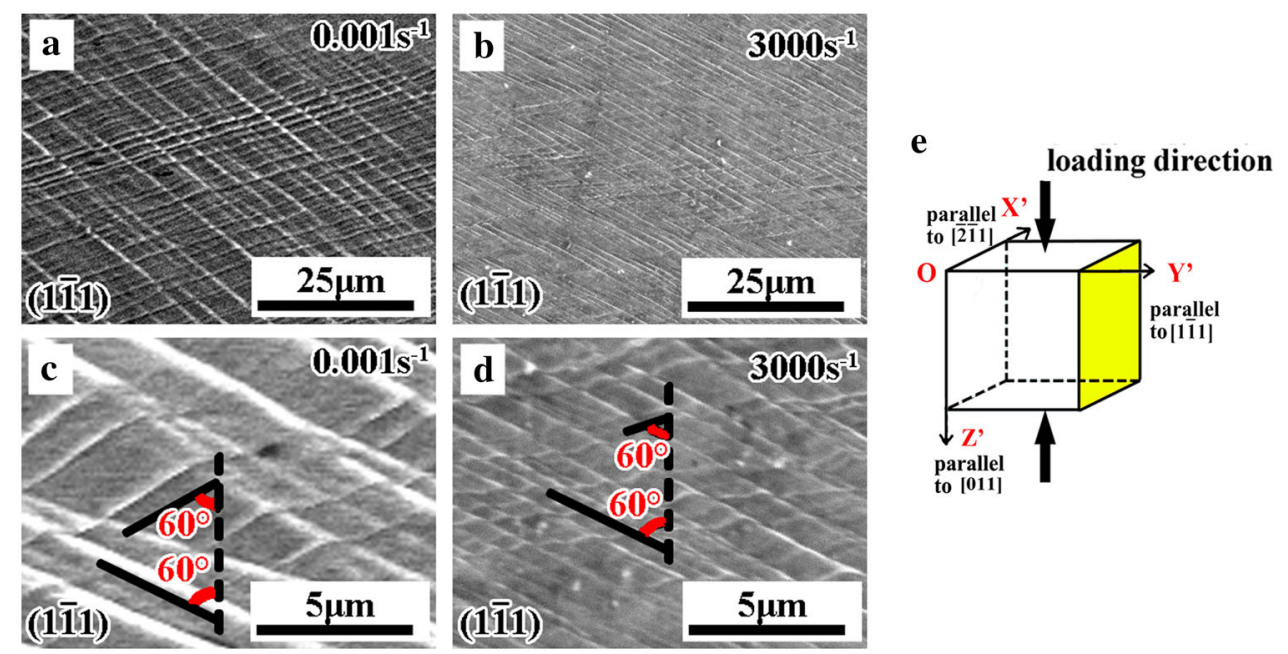

Fig. 14 SEM photograph of slip bands on (1111) side surface. a SEM micrograph of slip bands on quasi-static deformed specimen; b SEM micrograph of slip bands on dynamic deformed specimen; $\mathbf{c}$ Enlarged SEM micrograph of slip bands on quasi-static deformed specimen and diagrammatic sketch of their direction; d Enlarged slip bands of dynamic deformed specimen and diagrammatic sketch of their direction; and e Illustration of the relationship between the loading direction and (11̄1) side surface 
observed. Figure 14 compares the appearance of slip bands on the side surface of both dynamic deformed sample and the quasi-static one. Although $\mathrm{Ni}$ single crystal deforms by the same slip systems, and dynamic and quasi-static compressions cause the same D-ratio of slip systems, the slip bands formed under dynamic loading condition are denser and straighter than that formed under quasi-static condition, and the average separation distance (about $1.2 \mu \mathrm{m}$ ) of slip bands formed under dynamic condition is half of that under quasi-static condition (about $0.6 \mu \mathrm{m}$ ). The denser slip bands formed under dynamic condition indicate that the formation of slip band is easier under high strain rate condition, which show qualitative agreement with cross-slip simulations made by Wang et al. [27]. In other words, higher strain rates tend to increase the rate of band formation, but not change their structure [28].

In order to study the strain rate effect, the dynamic and quasi-static compression mechanical properties were investigated. Figure 15 shows the dynamic and quasi-static stress-strain curves of $\mathrm{Ni}$ single crystals, and it can be observed that Ni single crystal exhibits a little higher flow stress under dynamic loading condition, indicating an unobvious strain rate effect. This unobvious strain rate effect can be attributed to the same slip systems and the same corresponding D-ratio under both dynamic and quasistatic loading condition. The slightly enhanced flow stress under dynamic compression condition can be attributed to the effect of strain rate on movement of dislocation: the Peierls-Nabarro stress is increased with the increasing strain rate, leading to a larger driving force under dynamic condition.

In conclusion, under dynamic and quasi-static compression condition, the activated slip systems and their corresponding D-ratio of Ni single crystal are the same, but

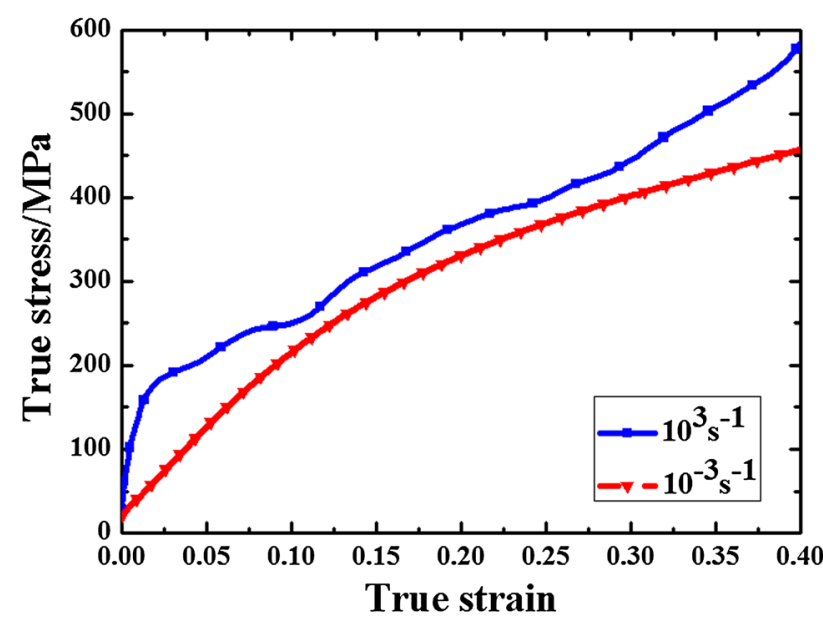

Fig. 15 Stress-strain curves of Ni single crystal under dynamic and quasi-static compression loading condition the density of slip bands on the side surface is different, indicating that higher strain rates tend to increase the rate of band formation. Moreover, Ni single crystal shows unobvious stain rate effect, which can be attributed to the same slip systems and same D-ratio of slip systems, while the larger driving force (the Peierls-Nabarro stress) of the dislocation under dynamic loading condition lead to a little higher flow stress.

Based on above results, it can be anticipated that, in a considerable range of strain rate (from $10^{-3}$ to $10^{3} \mathrm{~s}^{-1}$ ) FCC Ni single crystal deforms via dislocation slip with the same slip systems and same D-ratio of slip systems. However, it does not mean that other FCC single crystals also have the same deformation mechanism under different strain rate. In the field of dynamic deformation of single crystals, there are still many questions need to be studied, such as the effect of temperature, loading direction and stress state on single crystals with different crystal structures subjected to higher strain rate loading. Deeper insights into the dynamic deformation mechanisms of single crystals are of great value to the analysis and prediction of deformation mechanisms and mechanical behaviors of polycrystalline materials.

\section{Conclusion}

FCC Ni single crystal was dynamically compressed along [011] with a strain of 0.4. Results show that the dynamic deformation process of FCC Ni single crystal is completed via dislocation slip. Based on the analysis of Schmid Factor, orientation of the slip bands and dimensions of the deformed sample, the slip systems of $\mathrm{Ni}$ single crystal activated under dynamic compression along [011] direction

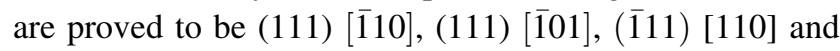
(111) [101] with a corresponding displacement ratio of nearly 1:3.8:3.8:1. Moreover, the slip systems and their corresponding D-ratio of $\mathrm{Ni}$ single crystal under dynamic loading are not changed with strain.

For comparison, quasi-static compression along the same direction with same strain was conducted to a same $\mathrm{Ni}$ single crystal. Results show that under quasi-static loading condition, the deformation mechanism of FCC Ni single crystal is also dislocation slip, and the slip systems and their corresponding D-ratio are the same as that under dynamic loading condition. Owing to the same slip systems and same corresponding D-ratio under both dynamic and quasi-static compression, FCC Ni single crystal shows unobvious stain rate effect. However, under dynamic loading condition, the slip bands on the side surface are denser than that under quasi-static condition, indicating that the formation of slip band is easier under high strain rate condition. 
Acknowledgments This research is supported by The National Science Foundation of China (No. 51571033). This work is supported in part by the National Natural Science Foundation of China under Grant No. 11521062. The authors gratefully acknowledge the support of the National Key Laboratory of Science and Technology on Material Under Shock and Impact.

\section{References}

1. Li P, Zhang ZF, Li XW, Li SX, Wang ZG (2009) Effect of orientation on the cyclic deformation behavior of silver single crystals: comparison with the behavior of copper and nickel single crystals. Acta Mater 57:4845-4854

2. Lychagin DV, Yu Tarasov S, Chumaevskii AV, Alfyorova EA (2015) Macrosegmentation and strain hardening stages in copper single crystals under compression. Int J Plast 69:36-53

3. Kumar MA, Mahesh S (2013) Subdivision and microtexture development in f.c.c. grains during plane strain compression. Int $\mathbf{J}$ Plast 44:95-110

4. Kim JY, Jang D, Greer JR (2009) Insight into the deformation behavior of niobium single crystals under uniaxial compression and tension at the nanoscale. Scr Mater 61:300-303

5. Liang Z, Seefeldt M, Verlinden B (2013) Three Nb single crystals processed by equal-channel angular pressing-Part II: mesoscopic bands. Acta Mater 61:4504-4511

6. Xu DS, Yang R, Li J, Chang JP, Wang H, Li D, Yip S (2006) Atomistic simulation of the influence of pressure on dislocation nucleation in bcc Mo. Comput Mater Sci 36:60-674

7. Tang Y, El-Awady JA (2014) Formation and slip of pyramidal dislocations in hexagonal close-packed magnesium single crystals. Acta Mater 71:319-332

8. Qin H, Jonas JJ, Yu H, Brodusch N, Gauvin R, Zhang X (2014) Initiation and accommodation of primary twins in high-purity titanium. Acta Mater. 71:293-305

9. Lychagin DV, Alfyorova EA, Starenchenko VA (2011) Effect of crystallogeometric states on the development of macrobands and deformation inhomogeneity in [-111] nickel single crystals. Phys Mesomech 14:66-78

10. Kumar MA, Mahesh S (2012) Banding in single crystals during plastic deformation. Int J Plast 36:15-33

11. Austin RA, McDowell DL (2012) Parameterization of a ratedependent model of shock-induced plasticity for copper, nickel, and aluminum. Int J Plast 32-33:134-154

12. Alankar A, Field DP, Raabe D (2014) Plastic anisotropy of electro-deposited pure a-iron with sharp crystallographic $<111>/ /$ texture in normal direction: analysis by an explicitly dislocation-based crystal plasticity model. Int $\mathbf{J}$ Plast $52: 18-32$
13. Lloyd JT, Clayton JD, Becker R, McDowell DL (2014) Simulation of shock wave propagation in single crystal and polycrystalline aluminum. Int J Plast 60:118-144

14. Galindo-Nava EI, Rivera-Díaz-del-Castillo PEJ (2014) Thermostastitical modelling of deformation twinning in HCP metals. Int J Plast 55:25-42

15. Rajulapati KV, Biener MM, Biener J (2010) Temperature dependence of the plastic flow behavior of tantalum. Philos Mag Lett 90:35-42

16. Wang L, Bei H, Li TL, Gao YF, George EG, Nieh TG (2011) Determining the activation energies and slip systems for dislocation nucleation in body-centered cubic Mo and face-centered cubic Ni single crystals. Scr Mater 65:182-197

17. Khan AS, Liu J, Yooh JW, Nambori R (2015) Strain rate effect of high purity aluminum single crystals: experiments and simulations. Int J Plast 67:39-52

18. Li QZ (2013) Microstructure and deformation mechanism of 0001 magnesium single crystal subjected to quasistatic and highstrain-rate compressive loadings. Mater Sci Eng A 568:96-101

19. Li ZL, Zhang DD, Su XL (2016) Role of as-cast dendritic microstructure in recrystallization of a Ni-based single crystal superalloy. J Alloy Compd 660:115-124

20. Yang YH, Yu JJ, Sun XF (2015) Temperature dependence of impact deformation behavior of single crystal Ni based superalloy. Mater Sci Technol 31:1860-1866

21. Westbrooke EF, Forero LE, Ebrahimi F (2005) Slip analysis in a Ni-base superalloy. Acta Mater 53:2137-2147

22. Hafez Haghighat SM, Eggeler G, Raabe D (2013) Effect of climb on dislocation mechanisms and creep rates in $\gamma^{\prime}$-strengthened $\mathrm{Ni}$ base superalloy single crystals: a discrete dislocation dynamics study. Acta Mater 61:3709-3723

23. Chen XF (1991) Saturated dislocation-structures of cyclic deformation ni [110] single crystals. Mater Sci Eng A 147:155-160

24. El-Awady JA, Uchic MD, Shade PA, Kim SL, Rao SR, Dimiduk DM, Woodward C (2013) Pre-straining effects on the power-law scaling of size-dependent strengthening in Ni single crystals. Scr Mater 68:207-210

25. Gurao NP, Kapoor R, Suwas S (2010) Effect of strain rate on evolution of the deformation microstructure and texture in polycrystalline copper and nickel. Metall Mater Trans A. 41A:2794-2804

26. Askeland DR, Phule PP (2008) Essentials of materials science and engineering, 2nd edn. Cengage Learning, Nelson

27. Wang ZQ, Beyerlein IJ, LeSar R (2008) Slip band formation and mobile dislocation density generation in high rate deformation of single fcc crystals. Philos Mag 88:1321-1343

28. Huang JC, Gray GT III (1989) Microband formation in shockloaded and quasi-statically deformed metals. Acta Metall $37: 3335-3347$ 